Ger J Exerc Sport Res 2021 · 51:222-231 https://doi.org/10.1007/s12662-021-00705-x Eingegangen: 27. August 2020

Angenommen: 10. Januar 2021

Online publiziert: 2. Februar 2021

๑) Der/die Autor(en) 2021
Denise Renninger ${ }^{1}$ (D) - David J. Sturm ${ }^{1}$ (D) $\cdot$ Claus Krieger $^{2} \cdot$ Yolanda Demetriou $^{1}$ (D)

${ }^{1}$ Fakultät für Sport- und Gesundheitswissenschaften, Technische Universität München, München, Deutschland

${ }^{2}$ Fakultät für Erziehungswissenschaft, Universität Hamburg, Hamburg, Deutschland

\title{
Förderung der körperlichen Aktivität von Mädchen im Sportunterricht
}

\section{Untersuchung der Implementierungs- qualität der CReActivity-Studie}

Die positive Wirkung körperlicher Aktivität auf die Entwicklung von Kindern und Jugendlichen ist ebenso unumstritten wie die Gesundheitsrisiken, die aus Bewegungsmangel resultieren (World Health Organization [WHO], 2010). Zwar manifestieren sich schwerwiegende Folgeerkrankungen meist erst im Erwachsenenalter, Studien zeigen aber, dass die in der Kindheit entwickelten Verhaltensweisen bezüglich körperlicher Aktivität über die Lebensspanne weitestgehend erhalten bleiben, was Konsequenzen für die aktuelle und langfristige Gesundheit nach sich zieht (Hallal, Victora, Azevedo, \& Wells, 2006; Telama, 2009). Die WHO empfiehlt Kindern und Jugendlichen daher täglich mindestens 60 min Bewegung mit moderater und intensiver Intensität (WHO, 2010). Obwohl Studien die Relevanz der Bewegungsempfehlungen hinsichtlich der Gesundheitsförderung bestätigen (Poitras et al., 2016; Strong et al., 2005), erreichen viele Kinder und Jugendliche in Deutschland - insbesondere Mädchen diese Empfehlungen nicht (Demetriou et al., 2019; Finger, Varnaccia, Borrmann, Lange, \& Mensink, 2018). Die Schule als organisatorischer Rahmen für Bewegungsförderungsmaßnahmen bietet die Möglichkeit, Aktivitätszeiten von Kindern und Jugendlichen zu er-

Verfügbarkeit von Daten und Material Auf Nachfrage können Transkripte der Interviews zur Verfügung gestellt werden. höhen. Reviews bestätigen zwar, dass schulbasierte Interventionen sowohl die Aktivität im Sportunterricht als auch im Alltag positiv beeinflussen können, die Effekte sind jedoch meist gering. Außerdem weisen diese Studien große methodische Heterogenität auf und werden teilweise mit einem hohen Risiko für systematische Fehler bewertet (Dobbins, Husson, DeCorby, \& LaRocca, 2013; Lonsdale et al., 2013).

Zudem bemängeln Lonsdale et al. (2013), dass die Implementierung vieler Studien nicht evaluiert wurde und somit die interne Validität nicht bewertet werden kann. Vor dem Hintergrund, dass die Programmimplementierung eine wichtige Determinante der Programmwirkung darstellt, ist dies besonders schwerwiegend (Durlak \& DuPre, 2008). Die Programmimplementierung bezieht sich hierbei auf die Umsetzung eines Programmes in der Realität, im Vergleich zu dessen theoretischer Konzeption (Durlak, 2016). Die Qualität dieser Implementierung lässt somit Rückschlüsse auf die Wirksamkeit oder Nichtwirksamkeit eines Programmes zu.

Generell erzielt ein Programm bessere Effekte, wenn das Implementierungsniveau höher ausfällt. Schlecht implementierte Programme hingegen können fälschlicherweise als uneffektiv bewertet werden, da sie nur schwache oder keine Effekte aufweisen (Durlak, 2016). Eine gute Implementierung schafft nicht nur bessere Voraussetzun- gen, um signifikante Interventionseffekte zu ermitteln, sondern erhöht auch den Nutzen für die Programmteilnehmer. Die Bewertung der Implementierung ist somit unerlässlich, um Interventionseffekte einordnen und Rückschlüsse auf die Programmwirksamkeit ziehen zu können. Hinsichtlich der Evaluation der Implementierung sportpädagogischer Programme bieten Sygusch, Bähr, Gerlach und Bund (2013) ein Rahmenkonzept, das die Untersuchung der Einhaltung der Programmvorgaben, der Häufigkeit der Implementierung, der Qualität der Programmweitergabe, des Sich-Involvieren der Teilnehmer sowie der Anwendbarkeit und Akzeptanz der Intervention empfiehlt. Im Rahmen der CReActivity-Studie wurde eine darauf basierende Implementierungsevaluation durchgeführt, welche Auskunft über das Implementierungsniveau der Studie geben soll. Die Cluster-randomisierte Studie verfolgt das Ziel, die körperliche Aktivität von Sechstklässlerinnen durch eine, auf der Selbstbestimmungstheorie (SBT; Deci \& Ryan, 2000) basierende, Intervention $\mathrm{zu}$ fördern (Demetriou \& Bachner, 2019). Laut der SBT werden Motivationsarten und deren Regulationen am Selbstbestimmungskontinuum von kontrolliert nach selbstbestimmt differenziert (Deci \& Ryan, 2002). Die sogenannte Internalisierung beschreibt den Prozess, bei dem ehemals extern motivierte (kontrollierte) Handlungen aufgrund von Veränderungen der Regu- 
lationsformen selbstbestimmter wahrgenommen werden und somit gewünschte Verhaltensweisen häufiger, intrinsisch motiviert, ausgeführt werden (Deci \& Ryan, 2002). Diese Förderung der Selbstbestimmung kann durch die Befriedigung der psychologischen Grundbedürfnisse Autonomie, Kompetenzerleben und soziale Eingebundenheit (AKS) erreicht werden. Auch im Sportunterricht kann durch ein bedürfnisorientiertes Unterrichtsklima die selbstbestimmte Motivation von Schülerinnen und Schülern gefördert und deren Aktivitätsverhalten beeinflusst werden (Deci \& Ryan, 2008; Reeve, 2009; Vasconcellos et al., 2019).

Im Rahmen der CReActivity-Studie sollten Lehrkräfte ein AKS-förderliches Unterrichtsklima herstellen, um die tägliche körperliche Aktivität der Schülerinnen zu erhöhen. Der verfolgte Ansatz geht auf das Konzept des selbstbestimmten Lernens zurück, welches in seinen Grundzügen auch bei der Konzeption des bayerischen Lehrplan PLUS berücksichtigt wurde. Die zugrundeliegende Kompetenzorientierung des Lehrplans wurde bei der Elaboration der AKS-förderlichen Unterrichtseinheiten ebenso einbezogen wie der Doppelauftrag des erziehenden Sportunterrichts. Dies wird in den bereitgestellten Stundenverlaufsplänen ${ }^{1}$ insbesondere an der regelmäßigen Aufforderung zur „bewegungsbezogenen Reflexion mit dem und über den eigenen Körper" deutlich, ohne dabei das Hauptaugenmerk des "Sich-Bewegens“ $\mathrm{zu}$ vernachlässigen (Wolters, Klinge, Klupsch-Sahlmann, \& Sinning, 2009). Hier spielt die Verhaltens- und Kommunikationsweise der Lehrkraft eine entscheidende Rolle (Ntoumanis, Quested, Reeve, \& Cheon, 2018). Im Sinne der SBT ermöglicht eine autonomieförderliche Lehrerin ebendiese Gelegenheit zur Reflexion und erweitert die Wahlund Entscheidungsmöglichkeiten wie die Schülerinnen ihren Sportunterricht erleben möchten, eingebettet in einer transparenten und begründeten Unterrichtsgestaltung (Reeve, 2016). Des Weiteren strebt die Lehrkraft zur Förde-

\footnotetext{
1 Das Studien-Manual sowie die Stundenverlaufspläne können auf Nachfrage bei den Autoren eingesehen werden.
}

rung des Kompetenzerlebens wahrhafte Erfolgserlebnisse der Schülerinnen an, die auch schon durch kleine Aufmerksamkeiten, wie positives Feedback, oder der Berücksichtigung von sportlichen Vorerfahrungen, begünstigt werden. Die bereits angeführte Zeit für Reflexion sollte insbesondere zur Förderung der sozialen Eingebundenheit bei gemeinsamen Ritualen genutzt werden, welche letztendlich nicht nur die Beziehung zwischen den Schülerinnen, sondern auch die Lehrer-Schüler-Beziehung auf ein vertrauensvolleres Niveau heben. Um diesem Anspruch gerecht zu werden, ohne dabei den Bezug zum Lehrplan zu verlieren, erhielten die Lehrerinnen der Interventionsgruppe eine Einführung in die SBT und ein darauf basierendes Unterrichtsmaterial bestehend aus 48 beispielhaften Stundenverlaufsplänen und Zusatzmaterial zu den Gegenstandsbereichen bzw. Handlungsfeldern des bayerischen Lehrplans für Basketball, Fußball, Gymnastik und Tanz, Gesundheit und Fitness, Schwimmen und Turnen. Die Lehrerinnen der Kontrollgruppe führten den Sportunterricht wie gewohnt durch.

Auf Basis der qualitativen Evaluation von Interviews mit den Lehrkräften sowie Fokusgruppen mit Schülerinnen soll die Fragestellung beantwortet werden: Wie erleben und deuten die Akteure die Intervention, insbesondere in Hinblick auf die Einhaltung der Programmvorgaben, die Häufigkeit der Implementierung, die Qualität der Programmweitergabe, das Sich-Involvieren der Teilnehmenden sowie die Anwendbarkeit und Akzeptanz des Programmes?

\section{Methodik}

\section{Stichprobe}

Alle acht Lehrerinnen der Interventionsgruppe nahmen an den Interviews teil. Pro Interventionsklasse wurden zudem vier bzw. fünf Schülerinnen ausgewählt $(n=41)$. Die Lehrerin wählte zwei Schülerinnen, die im Sportunterricht meist motiviert waren und zwei Schülerinnen, die weniger Begeisterung zeigten. Die Ethikkommission der Technischen Universität München sowie das Bayerische
Staatsministerium für Unterricht und Kultus bewilligte die Durchführung der Studie. Schulleitung und Elternbeirat sowie teilnehmende Lehrerinnen gaben der Durchführung ihr Einverständnis. Eltern bzw. Erziehungsberechtigte sowie Schülerinnen stimmten nach schriftlicher Aufklärung der Datenerhebung und -verarbeitung zu. Vor jedem Interview wurde ein mündliches Einverständnis zur Teilnahme eingeholt.

\section{Leitfadenorientierte Interviews}

Zur Leitfadenkonzeption wurden MindMaps erstellt, welche alle relevanten Themengebiete umfassten. Dabei wurden die Orientierungspunkte zur Evaluation der Durchführung sportpädagogischer Programme berücksichtigt (Sygusch et al., 2013), die sich auf die Einhaltung der Programmvorgaben, die Häufigkeit der Implementierung, die Qualität der Programmweitergabe, das Sich-Involvieren der Teilnehmer sowie die Anwendbarkeit und Akzeptanz der Intervention beziehen. So ergaben sich für die Interviews mit den Lehrerinnen Themenblöcke zur korrekten Durchführung bzw. zu den Veränderungen der Vorgaben von CReActivity, zur Bewertung der Durchführbarkeit der Intervention und zu Verbesserungsvorschlägen. Die Themen der Schülerinnen-Interviews beinhalteten das Erleben von AKS im Sportunterricht, den Sportunterricht im Allgemeinen und die körperliche Aktivität in der Freizeit. Um Verständlichkeit und Relevanz der Fragen zu prüfen, wurden Probeinterviews durchgeführt. Zudem wurden die Leitfäden während der Datenerhebung kontinuierlich weiterentwickelt, um detaillierte Informationen $\mathrm{zu}$ erhalten.

\section{Interviewdurchführung}

Die Interviewdurchführung erfolgte während des Posttests der CReActivityStudie, im Anschluss an die Interventionsperiode. Vor Beginn wurden Ablauf und Hintergrund des Interviews erklärt und Fragen der Teilnehmer besprochen. Die Dauer der Lehrerinnen-Interviews betrug durchschnittlich 15 min (Spannweite: $10 \mathrm{~min}$; Min: $12 \mathrm{~min}$; Max: $22 \mathrm{~min}$ ). 
Die Wahl des Ortes für die SchülerinnenGruppeninterviews traf die Lehrkraft der jeweiligen Klasse. Die Dauer der Gruppeninterviews betrug durchschnittlich 19 min und variierte deutlich (Spannweite: $14 \mathrm{~min}$, Min: $12 \mathrm{~min}$, Max: $26 \mathrm{~min}$ ).

\section{Datenanalyse}

Alle Interviews wurden aufgenommen, wörtlich transkribiert und nach den Schritten der thematischen Analyse (TA, „thematic analysis") von Braun und Clarke (2006) in einem deduktiv-induktiven Prozess ausgewertet. Zunächst wurde jedes Interview hinsichtlich der Aspekte der Implementierungsqualität deduktiv untersucht und entsprechende Textstellen gekennzeichnet. Die LehrerinnenInterviews wurden nach allen Kriterien analysiert, um aussagekräftige Schlüsse über die Implementierungsqualität ziehen zu können (Naylor et al., 2015). Da CReActivity die Art der Vermittlung von Unterrichtskomponenten fokussierte, wurden in der Analyse zusätzlich die Interviews mit den Schülerinnen berücksichtigt. Diese wurden hinsichtlich Qualität der Programmweitergabe und Sich-Involvieren aus Teilnehmersicht untersucht. Innerhalb dieses deduktiven Rahmens wurde anschließend induktiv kodiert, um eine möglichst datengesteuerte Bottom-up-Analyse zu ermöglichen. Codes, die einen ähnlichen Inhalt beschrieben, wurden im Anschluss in Kategorien zusammengefasst, welche wiederum am Material geprüft wurden. Der Prozess der Kategorienbildung gestaltete sich somit iterativ, da Codes und Kategorien stetig weiterentwickelt wurden.

\section{Ergebnisse}

Aus der Analyse der Interviews ergaben sich neun Kategorien, die im Folgenden beispielhaft illustriert und interpretiert werden. Tab. 1 bietet einen Überblick über die Kategorien, die aus den Interviews mit Lehrerinnen und Schülerinnen gewonnen werden konnten.

Ger J Exerc Sport Res 2021 · 51:222-231 https://doi.org/10.1007/s12662-021-00705-x

(c) Der/die Autor(en) 2021

D. Renninger · D. J. Sturm · C. Krieger · Y. Demetriou

\section{Förderung der körperlichen Aktivität von Mädchen im Sportunterricht. Untersuchung der Implementierungsqualität der CReActivity-Studie}

\section{Zusammenfassung}

Die Förderung eines aktiven und gesunden Lebensstils ist eine wichtige Aufgabe der Schule und des Sportunterrichts. Interventionsprogramme in diesem Setting besitzen zudem einen hohen Stellenwert zur Förderung körperlicher Aktivität von Kindern und Jugendlichen. Um die Wirksamkeit dieser Interventionsprogramme zu erfassen, ist eine umfangreiche Evaluation notwendig. Ziel der vorliegenden Untersuchung ist die qualitative Bewertung der Implementierungsqualität des CReActivity-Interventionsprogrammes, welches, basierend auf der Selbstbestimmungstheorie, die Förderung der körperlichen Aktivität von Schülerinnen durch den Sportunterricht zum Ziel hat. Zu diesem Zweck wurden leitfadenorientierte Interviews mit Lehrerinnen und Schülerinnen der Interventionsklassen des Projekts durchgeführt. Anschließend wurden die Interviews mithilfe der thematischen Analyse deduktivinduktiv untersucht, um Erkenntnisse zu gewinnen, wie die Teilnehmerinnen die Intervention erleben und deuten. Die
Analyse der Interviews der Lehrerinnen ergab insgesamt acht Themen, in denen die Einhaltung der Programmvorgaben, die Häufigkeit der Implementierung, die Qualität der Programmweitergabe, das Sich-Involvieren der Teilnehmer, die Anwendbarkeit der Intervention und die Akzeptanz aufgegriffen werden. Aus den Interviews mit den Schülerinnen konnten Informationen hinsichtlich der Qualität der Programmweitergabe und des SichInvolvierens der Teilnehmer gewonnen werden. Insgesamt lassen die Ergebnisse eine von den Lehrerinnen wahrgenommene Wirksamkeit der Maßnahme erkennen, die auf eine hohe Implementierungsqualität verweist. Dennoch lassen die Ergebnisse eine schwankende Implementierungsqualität der einzelnen Lehrerinnen erkennen.

\section{Schlüsselwörter}

Programmimplementierung · Programmevaluation · Schulbasierte Gesundheitsförderung . Thematische Analyse - Leitfadeninterviews

\section{Promoting girls' physical activity in physical education. Evaluation of the implementation quality of the CReActivity study}

\section{Abstract}

The promotion of an active and healthy lifestyle is a central mission of the school setting and physical education lessons. Intervention programs in this setting have a high priority in promoting physical activity in children and adolescents. In order to determine the effectiveness of an intervention program, a comprehensive evaluation is essential. The aim of the present study is the qualitative evaluation of the implementation quality of the CReActivity study, which is based on the self-determination theory and aimed to promote girls' physical activity levels through physical education. For this purpose, guided interviews were carried out with female teachers and students of the intervention classes. Subsequently, interviews were analyzed by means of deductive-inductive thematic analysis to gain insight into how students and teachers experience and interpret the program. The analysis of the teacher interviews revealed eight themes regarding program adherence, frequency of implementation, quality of delivery, participant responsiveness, applicability and acceptance of the intervention. Student interviews provided information regarding quality of delivery and participant responsiveness. Overall, the results indicate a perceived effectiveness of the intervention by the teachers, which indicates a high quality implementation. Nevertheless, the results suggest different levels of implementation quality between teachers, which contribute to an inconsistent picture of the implementation quality.

\section{Keywords}

Program implementation · Program evaluation -School-based health promotion . Thematic analysis - Guided interviews 


\begin{tabular}{|c|c|c|}
\hline $\begin{array}{l}\text { Aspekt der Implemen- } \\
\text { tierungsqualität }\end{array}$ & Sicht der Sportlehrerinnen & $\begin{array}{l}\text { Sicht der Schülerin- } \\
\text { nen }\end{array}$ \\
\hline $\begin{array}{l}\text { Einhaltung der Pro- } \\
\text { grammvorgaben }\end{array}$ & $\begin{array}{l}\text { 1. Orientierungsgrundlage und notwendige } \\
\text { Variation der konzeptionellen Vorgaben }\end{array}$ & - \\
\hline $\begin{array}{l}\text { Häufigkeit der Imple- } \\
\text { mentierung }\end{array}$ & 2. Limitierender Rahmen des Schulsports & - \\
\hline $\begin{array}{l}\text { Qualität der Programm- } \\
\text { weitergabe }\end{array}$ & 3. Studienteilnahme als Motivation & \multirow[t]{2}{*}{$\begin{array}{l}\text { 4. Abhängigkeit von } \\
\text { Lehrerengagement }\end{array}$} \\
\hline $\begin{array}{l}\text { Sich-Involvieren der } \\
\text { Teilnehmer }\end{array}$ & $\begin{array}{l}\text { 5. (Überraschend) hohes Engagement und akti- } \\
\text { ves Einlassen }\end{array}$ & \\
\hline Anwendbarkeit & $\begin{array}{l}\text { 6. Angemessenheit der Inhalte } \\
\text { 7. Passung zu den eigenen Voraussetzungen/ } \\
\text { Ressourcen } \\
\text { 8. Ausrichtung auf (unrealistische) Optimalbedin- } \\
\text { gungen }\end{array}$ & - \\
\hline Akzeptanz & 9. Fachlich sinnvolle Bereicherung & - \\
\hline
\end{tabular}

\section{Orientierungsgrundlage und notwendige Variation der konzeptionellen Vorgaben}

Diese Kategorie verweist auf ein wiederkehrendes Spannungsfeld der vorgegebenen Planungsgrundlage und der notwendigen Flexibilität im Sportunterricht: Die Lehrerinnen verwendeten die bereitgestellten Stundenverlaufspläne, was sich in den Interviews beispielsweise daran zeigt, dass sie sich auf konkrete Übungsbeispiele bezogen, um Situationen zu beschreiben, in denen AKS der Schülerinnen gefördert wurde. Andererseits erklärten die Lehrkräfte, dass sie bei der Unterrichtsplanung und -umsetzung von den konzeptionellen Vorgaben abweichen mussten, um die Durchführung zu ermöglichen. Begründet wurde dies u.a. mit dem hohen Umfang der Stunden und der Rücksichtnahme auf die Bedürfnisse der Schülerinnen. Meist beschränkten sich die Lehrerinnen daher auf einzelne Komponenten bzw. Sequenzen aus den jeweiligen Handlungsfeldern. Insofern durchziehen auch Bezüge zwischen dem regulären und dem veränderten Sportunterricht die Interviews.

\section{Beispiele}

A. Also wir versuchen das [Fördern von AKS im Sportunterricht] natürlich im normalen Sportunterricht auch, aber klar, wenn man diese drei Bausteine sich setzt, dann ist das natürlich wieder was Anderes. (Lehrerin 6)
B. Wenn, dann war es, dass ich ein Themengebiet zum Beispiel Fußball rausgesucht habe, dass die (...) Mädchen am wenigsten interessiert hat. Dann war es wahnsinnig schwer, die Stunden durchzuziehen, und da hab' ich dann die Stunden verändern müssen. (...) das hätte sonst nicht geklappt. (Lehrerin 1)

Die erste Interviewsequenz verdeutlicht insbesondere, dass es sich bei der Intervention für die befragte Lehrkraft um „was Anderes“, im Sinne von etwas Besonderes im Vergleich zum „normalen Sportunterricht" handelt. Gegenüber der alltäglichen, selbstständigen Planung der Unterrichtsstunden werden hier ausgearbeitete Bausteine serviert, die übernommen werden können. Lehrerin 1 weist hingegen darauf hin, dass der Durchführung von ausgearbeiteten Themengebieten Grenzen gesetzt sind - hier ist Variation bzw. Modifikation durch (unterstelltes oder wahrgenommenes) schülerinnenbezogenes Desinteresse am Fußball notwendig.

\section{Limitierender Rahmen des Schulsports}

Diese Kategorie beschreibt die Abhängigkeit der Implementierungshäufigkeit von den Gegebenheiten der Schule. Die Lehrerinnen konnten die Intervention ausschließlich im Rahmen verfügbarer Sportstunden und Sportstätten implementieren und mussten die curricularen
Vorgaben einhalten. Meist konnten sich die Lehrerinnen mittels gezielter Auswahl der bereitgestellten Handlungsfelder diesen Vorgaben anpassen und Interventionsinhalte in den Sportunterricht integrieren. Dennoch beschreiben einige Lehrerinnen Zweifel an der langfristigen Wirksamkeit der Intervention aufgrund der eingeschränkten Umsetzungshäufigkeit durch diese strukturellen Vorgaben.

\section{Beispiele}

A. Das ist immer so: Also ich mache immer diese Handlungsfelder zu dieser Zeit. Das hat bei uns auch so ein bisschen mit den Außenanlagen und sowas zu tun, ähm, wir haben gar keine Möglichkeit/wir müssen zum Sportverein laufen. Das geht im Sommer, aber wir sind jetzt hier in den Hallen, und die Halle wird dann für die Abschlussprüfung hergenommen und so. Deswegen müssen wir die Noten auch vorher machen. Deswegen fange ich immer (...) so damit an. (Lehrerin 6)

B. Ähm, ich glaube in den Stunden danach direkt: Ja. Aber längerfristig die Kinder an den Sport zu binden, dass das man längerfristige Veränderungen erreicht, bräuchten wir mehr Sport mit den Kindern, ja. Ich habe die nur einmal die Woche zwei Stunden. Das ist wirklich wenig. Aber danach, nach dem Unterricht war immer glaub' ich der Effekt bei den meisten positiv. (Lehrerin 1)

In der ersten Interviewsequenz spricht Lehrerin 6 die räumlichen Gegebenheiten an, in deren Rahmen der Sportunterricht gestaltet und die Intervention vermittelt werden kann. In der zweiten Interviewsequenz beschreibt Lehrerin 1 die Abhängigkeit der Implementierungshäufigkeit von den verfügbaren Sportunterrichtsstunden und bewertet diese Häufigkeit von einmal die Woche zwei Stunden als unzureichend, um langfristige Effekte erzielen zu können.

\section{Studienteilnahme als Motivation}

Diese Kategorie greift die insgesamt hohe Motivation der Lehrerinnen zur Teilnahme an der Studie auf, die hauptsäch- 
lich von dem Interesse zur Teilnahme an einer Intervention geprägt war. Teilweise erhoffen sich die Lehrerinnen dadurch einen Erfahrungsgewinn und fachliche Weiterbildung, andere hoffen damit zu vernünftigen, brauchbaren Studienergebnissen beizutragen.

\section{Beispiele}

A. Ich habe noch nie bei so einer Intervention mitgemacht. Ich finde das jetzt schon mal ganz gut. (...) Auch fachlich hat es mich vorwärtsgebracht. Ich bin gespannt jetzt, wie es mit der Auswertung ist. (Lehrerin 1)

B. Also ich möchte mich einfach bedanken für diese Möglichkeit, dass wir (...) auch diesen Einblick in diese Arbeit haben konnten. (Lehrerin 3)

Lehrerin 1 stellt im ersten Interviewausschnitt die Intervention als persönlich bereichernde und spannende Erfahrung dar, die Entwicklungs- und Innovationspotenzial verspricht. Die Erwartungen an die Studienteilnahme werden damit erfüllt und hinsichtlich der ausstehenden Studienergebnisse erweitert. Auch der Dank von Lehrerin 3, einen Einblick in „diese Arbeit“ zu erlangen, verdeutlicht, dass eine hohe Motivation zur Studienteilnahme bestand und der erzielte Erfahrungsgewinn die Teilnahme rechtfertigt.

\section{Abhängigkeit vom Lehrer- engagement}

Diese Kategorie erfasst die Relevanz des Lehrerengagements im Rahmen der Interventionsdurchführung aus Schülerinnensicht. Die Bewertung der Lehrenden-Lernenden-Interaktion war aus deren Perspektive stark vom Lehrerengagement geprägt. Positiv empfanden die Schülerinnen individuelle Unterstützung und Interesse seitens der Lehrkraft. Abhängig von diesem Engagement beschreiben die Schülerinnen mehrere Gründe für die Teilnahme am Unterricht. Bei einem als gut eingeschätzten Lehrerengagement gaben die Schülerinnen vor allem Spaß als Beweggrund an. Außerdem schilderten es die Schülerinnen als motivierend, wenn das Selbstvertrauen gestärkt und Fähigkei- ten von der Lehrerin unterstützt wurden. Zudem involvierten sich die Schülerinnen eher in unbeliebten Übungen, wenn die Lehrkraft die Übungsauswahl vorher begründete. Negativ fielen den Schülerinnen hingegen unfaire oder ungleiche Behandlungen sowie Desinteresse auf. In diesen Fällen zeigte sich die Angst vor negativen Konsequenzen, wie zum Beispiel schlechten Noten oder das Laut werden, als Grund, am Sportunterricht teilzunehmen.

\section{Beispiele}

A. Schülerin (S) 1: Aber bei Fußball hat sie uns gesagt, weil wir uns auch also trauen sollen zu sagen, dass auch Mädchen Fußball spielen können, nicht nur Jungs. (...)

S2: Ich fand das sehr schön, dass sie das gesagt hat, dass auch Mädchen Fußball machen können. (Klasse 4)

B. S1: Also ich oder die Lehrerin geben uns ja auch Mut, dass wir es schaffen können, und dann habe ich ja auch selber das Gefühl, dass ich es schaffe, und dann probiere ich es halt aus. S2: Man hat aber auch manchmal, wenn es für einen zu schwer ist, das Gefühl, dass man es gar nicht kann. Interviewleitung: Okay. Was macht dann die Frau (Lehrerin 1)?

S2: Ja dann gibt sie uns Mut und sagt, dass wir es schaffen und ja. Ist eigentlich ganz gut. (Klasse 4)

C. S1: Wir hatten neulich auch so einen Vorfall mit einem Mädchen aus unserer Klasse und wir waren Joggen und dieses Mädchen hat halt ein bisschen Asthma (...) und konnte dann nicht mehr und hat/ konnte nicht mehr so gut atmen und dann sind die zur Frau (Lehrerin 5) gegangen und haben gesagt: Ja, sie hat halt Atemprobleme gerade, kann sie sich kurz ausruhen? Dann hat die Frau (Lehrerin 5) nur zu ihr gesagt: Kuck nicht so, also würdest du gleich heulen! Und ist weiter gejoggt. (...) S2: Ja, ich als Sportlehrer würde das überhaupt nicht machen, ich würde sofort stehenbleiben und mich auch um das Kind kümmern.

S1: Und dann stand sie halt alleine da und die anderen waren schon um den Block. (...) Und sie durfte auch nicht mit der Freundin dastehen, die Freundin musste dann weiter rennen und sie stand dann ganz alleine da. (...)

S3: Sie droht einem dann auch immer so, dass man dann eine Sechs eingetragen kriegt, und wenn man dann einmal irgendwie redet oder so, dann sagt sie auch manchmal: mündliche Sechs! Und dann muss man raus oder so und jetzt also zum Beispiel: (...) halt das Mädchen mit Asthma dastand und die Freundin daneben, hat sie gleich zu ihrer Freundin gesagt: Ja, mündliche Sechs, du kommst jetzt mit. Oder so. (Klasse 2)

Die Interviewausschnitte verweisen auf das Erleben eines positiv-förderlichen wie auch negativ-diffamierenden Lehrerengagements. Im ersten Interviewausschnitt beschreiben die Schülerinnen, wie eine potenziell bedrohliche und verunsichernde Situation (das bevorstehende Fußballspielen) von der Lehrerin produktiv aufgelöst und die Situation dadurch entdramatisiert wird. Beispiel B greift das Lehrerengagement hinsichtlich Empathie und Selbstwertstärkung auf; die Lehrerin vermittelt und ermöglicht den Schülerinnen ein Gefühl der Sicherheit und des Glaubens an sich und die eigenen Fähigkeiten. Dies nennt eine Schülerin als Grund für das weitere Ausprobieren und des Involvierens in den Sportunterricht. Dem gegenüber steht der Interviewausschnitt $\mathrm{C}$, in dem die Schülerinnen dieses Verständnis seitens der Lehrkraft vermissen und stattdessen deren Diffamierungen ausgesetzt sind. Sie erhoffen sich Unterstützung, fühlen sich jedoch abgewiesen, verunsichert und in ihrem Selbstwert geschwächt. In weiterer Folge nehmen die Schülerinnen nur am Sportunterricht teil, um negativen Konsequenzen (der mündlichen Sechs) zu entgehen.

\section{5. (Überraschend) hohes Engagement und aktives Einlassen der Schülerinnen}

Die Kategorie umfasst die Annahme der Lehrerinnen, dass die Schülerinnen die angebotenen Möglichkeiten zum AKS- 
Erleben wahrnahmen und gerne am Unterricht teilnahmen. Dies zeigte sich laut Lehrerinnen in der Bereitschaft der Schülerinnen, beispielsweise Hausaufgaben zu erledigen und dem expliziten Nachfragen einiger Übungen. Dennoch lassen sich klassen- und handlungsfeldabhängige Unterschiede erkennen: Beispielsweise äußerte eine Lehrerin einen negativen Eindruck hinsichtlich der Fitness-Stunden. Eine andere Lehrerin erklärte hingegen, dass eben diese Fitness-Stunde quasi ein Selbstläufer war.

\section{Beispiele}

A. Also speziell in dem Bereich Basketball und (...) Turnen, ähm, hat es sich so ergeben, wenn sie in einer Gruppenarbeit arbeiten konnten und in einem Parcour arbeiten, den sie selber erstellen durften, mit den Möglichkeiten, die jedes Kind hat, sind die erfolgreichsten Dinge daraus entstanden. (Lehrerin 5)

B. Ja, also ich hab' ähm das Turnen gemacht, den Handstand vor allem Dingen. Und ja, da war ja die Übung auch, ähm, zu Hause, dass man das üben soll bis zum nächsten Mal. Und ich war sehr überrascht, dass die Schüler das wirklich selber zu Hause geübt haben. Und die waren dann so eifrig, natürlich nicht alle, aber einige, die das dann wirklich Mal reibungslos zeigen konnten, und sie haben vorher noch nie einen Handstand gemacht. Also weil sie immer sich nicht getraut haben und durch diesen Wettbewerb, da haben auch einige das mit ihren Geschwistern gemacht, haben die das super gut draufgehabt. Also, da waren sie sehr eifrig dabei, da war ich sehr überrascht. (Lehrerin 2)

Im ersten Interviewausschnitt schätzt Lehrerin 5 die angewandten Strategien zur AKS-Förderung (Gruppenarbeiten und Erstellen eines Parcours) als erfolgreich ein, da sich die Schülerinnen, im Rahmen individueller Möglichkeiten, auf die jeweiligen Strategien einließen. In der zweiten Sequenz verweist Lehrerin 2 auf Übungen, die zu Hause wiederholt werden sollten. Dabei wird eine unerwartete Bereitschaft und ho- hes Engagement der Schülerinnen, diese Hausaufgaben durchzuführen und teilweise die Geschwister miteinzubeziehen, erkannt.

\section{Angemessenheit der Inhalte}

Diese Kategorie umfasst die Angemessenheit der Intervention für die Zielgruppe. Aus Sicht der Lehrerinnen stellen die Übungen für die Schülerinnen weder eine Über- noch Unterforderung dar. Bei auftretenden Problemen konnten die Lehrerinnen unterstützend einwirken und so sicherstellen, dass die Schülerinnen die Übungen umsetzen konnten. Des Weiteren empfanden die Lehrerinnen die Interventionsinhalte zum Lehrplan passend.

\section{Beispiel}

A. Und da war es schon so, dass da, die Voraussetzungen sind ja immer sehr unterschiedlich. Klassen sind immer extrem heterogen und gerade (...) [dieses Schuljahr] habe ich sehr, sehr stark divergierende Klassen in der Leistung. Und, ähm, da hatte ich eben schon das Gefühl, dass jeder so ein bisschen, ähm, zum Zug kommt. Genau mit dem (...), was sie kann. Also gerade die turnerischen Elemente, ähm, gefragt, und da hat sich jede so eingebracht, wie sie eben das Gefühlt hat, da bin ich jetzt an der richtigen Position. (Lehrerin 3)

Die Lehrerin öffnet zunächst den Kontext und die Problematik heterogener Leistungsvoraussetzungen von Schülerinnen, um dann die (individuelle) Passung der durchgeführten Interventionsinhalte zu betonen. Die Lehrerin verdeutlicht dadurch, dass die konzipierten Unterrichtsstunden eine Teilnahme, unabhängig vom individuellen Leistungsniveau, ermöglichten.

\section{Passung zu den eigenen Ressourcen}

Diese Kategorie greift die Anwendbarkeit der Intervention durch die Lehrerinnen auf. Die Interventionsumsetzung bereitete den Lehrerinnen kaum Probleme, da die Studieninformationen als verständlich und die Vorbereitungen als pas- send empfunden wurden. Etwaige Probleme konnten nach Rücksprache mit der Studienleitung oder Kollegen gelöst werden. Dies förderte ein zunehmend besseres Gefühl und Verständnis für die Umsetzung im Interventionsverlauf, welches laut Lehrerinnen durch ihre praktische Erfahrung essenziell bereichert wurde.

\section{Beispiele}

A. Also ich hatte ja die Informationen in dem Ordner auch noch. Das heißt, ich bin ja jemand, ich habe immer ganz gerne, ähm, etwas schwarz auf weiß. Also da habe ich mich wahnsinnig gut informiert gefühlt, und ansonsten habe ich den D. zwei-, dreimal angerufen und das war sehr unproblematisch. Fand ich total gut. (Lehrerin 1)

B. Das hat ja im Groben hat es gepasst. Man könnte noch ein bisschen mehr noch mal so heraus/also ich hab' dann erst so beim/ich bin mir sicher, er hat das alles gesagt, aber das ist dann erst so mit der Zeit. (...) am Anfang so huh und das und das, genau. (...) Es ist halt am Anfang tatsächlich sehr viel und dann. Ich hab' zwei, drei Stunden gebraucht, dann habe ich mich mit meiner Kollegin besprochen und ist uns das so: ach darum geht es. (Lehrerin 8)

Die Betonung von Lehrerin 1, sich aufgrund der zur Verfügung gestellten Informationen „wahnsinnig gut informiert gefühlt" zu haben und die Beschreibung der eigenen Vorlieben „etwas gerne schwarz auf weiß" zu haben, legen nahe, dass die Lehrerin die Art der Informationsvermittlung als auf ihre persönlichen Ressourcen abgestimmt empfand. Die zweite Interviewsequenz verweist auf eine anfangs auftretende Unsicherheit der Lehrerin, die jedoch mit Hilfe einer Kollegin, die ebenfalls an CReActivity teilnahm, schnell und effektiv überwunden werden konnte. Hier zeigt sich ebenfalls die Passung der Intervention zu den Ressourcen der Lehrkraft. 


\section{Ausrichtung auf (unrealistische) Optimalbedingungen}

Die Kategorie verweist auf die Problematik der Interventionsausrichtung auf optimale Rahmenbedingungen des Sportunterrichts. Die Lehrerinnen erklärten, dass der inhaltliche Umfang der Interventionsstunden den zeitlichen Rahmen des Sportunterrichts überstieg und die Konzeption viele praktische Aspekte wie Klassengröße, verfügbares Material und individuelle Klassensituationen vernachlässigte. Dennoch war es den Lehrerinnen möglich, durch Anpassungen, Interventionsstunden durchzuführen.

\section{Beispiele}

A. Ähm, was mir aufgefallen ist von den ganzen Sequenzen her, ist das ganze Konzept für weniger Schüler mit reichlich Material. Aber, wenn du über 30 Schüler in nur einer Halle hast, ist das sehr, sehr schwierig. Von den Gruppen her, Gruppengröße und Material. Dass wirklich auch Platz ist, vom Platz her. (Lehrerin 7)

B. Alles vom Zeitlichen, war meine Erfahrung eigentlich egal welche Stunde, wenn ich das mit erklären/und es kommen ja auch Fragen immer von den Schülern (...) das sind alles so zeitliche Sachen, die sind nicht miteingeplant. Und wenn man komplett vielleicht eineinhalb Stunden hätte, aber die hat man nie. (...) [Dann] sind die ganzen Sequenzen (...) nicht zu schaffen. (Lehrerin 7)

In beiden Interviewsequenzen sind Grenzen der Machbarkeit und Wirksamkeit der Intervention benannt. Die Lehrerin beschreibt praktische Schwierigkeiten, da zu wenig Material, Platz und Zeit verfügbar war, um die Interventionsstunden entsprechend der Konzeption durchzuführen. Für sie steht die Konzeption im Konflikt oder gar Widerspruch zu den Alltagslogiken des Sportunterrichts.

\section{Fachlich sinnvolle Bereicherung}

Diese Kategorie bezieht sich auf die generelle Akzeptanz der Intervention und beschreibt die inhaltliche Sinnhaftigkeit der Maßnahme aus Sicht der Lehrerinnen. Diese zeigt sich unter anderem in der empfundenen Übereinstimmung der Interventionsziele mit den persönlichen Zielen der Lehrerinnen. Nach Einschätzung der Lehrerinnen ergibt sich aus der Intervention eine Bereicherung, da durch den Erwerb neuer pädagogischer Kompetenzen indirekt auch die Schülerinnen profitieren. Zudem verdeutlicht die Intention der Lehrerinnen, das bereitgestellte Material weiter $\mathrm{zu}$ verwenden und die Studieninhalte fortzuführen, den Nutzen der Studie.

\section{Beispiele}

A. Lehrerin: Und das ist natürlich genau das was wir wollen im Sport. Das sie merken, sie können was.

Interviewleitung: Also geht das sozusagen auch mit Ihren Zielen überein?

Lehrerin: Absolut. (...) Mein Ziel ist es, dass sie merken, ähm, du musst verschiedene Dinge ausprobieren. Ich versuche, dass sie möglichst viel ausprobieren und dass sie dann einen Erfolg haben. Und möglichst dann irgendwo hängen bleiben im Sport. (Lehrerin 1)

B. Eine [Schülerin] ist mir besonders aufgefallen. Die hat sich (...) beim Turnen nicht so getraut und dann hab' ich eine besondere Stunde aus dem Skript gemacht und danach war die total happy und hat gesagt: Mensch und ich konnte das. Das war die schönste Sportstunde, die ich je hatte. Also das hat mich sehr gefreut. Fand ich toll. (Lehrerin 1)

Lehrerin 1 betont, dass es "genau das ist", was sie mit dem Sportunterricht erreichen möchte. Diese Kongruenz der Ziele verdeutlicht die Sinnhaftigkeit, die sie der Intervention zuschreibt. In Sequenz B berichtet die Lehrerin von der Freude, durch Zuhilfenahme der bereitgestellten Materialien, die Ängste einer Schülerin überwinden zu können. Die Betonung des Einsatzes einer „besonderen Stunde“, verdeutlicht die als sinnvoll empfundene Bereicherung der Interventionsinhalte, die laut Lehrerin maßgeb- lich zum Erfolg der Schülerinnen beigetrugen.

\section{Diskussion}

Diese Untersuchung evaluiert die Implementierungsqualität der Cluster-randomisierten Interventionsstudie CReActivity, welche beabsichtigte, die körperliche Aktivität von Mädchen durch eine theoriebasierte Modifizierung des Sportunterrichts zu fördern. Die Analyse von leitfadenorientierten Interviews offenbarte die Wahrnehmung und Deutung der Intervention von Lehrerinnen und Schülerinnen. Im Folgenden werden die Ergebnisse und deren Einfluss auf die Implementierungsqualität diskutiert.

Die Analyse zur Einhaltung der Programmvorgaben verweist auf die Orientierung der Lehrerinnen bei den Unterrichtsvorbereitungen am Skript, welche das theoretische Verständnis der Lehrerinnen für Interventionsinhalte und -methoden begünstigte. Aufgrund der zeitlichen und räumlichen Dynamik des Sportunterrichts mussten die Lehrerinnen die Vorgaben verändern. Dabei wurden Unterrichtsstunden gekürzt, aber auch Kernkomponenten der AKS-Förderung abgewandelt. Adaptionen konzeptioneller Vorgaben werden auch in anderen Interventionsstudien berichtet und können positive Auswirkungen auf die Programmergebnisse erzielen (Domitrovich et al., 2008), jedoch nur, wenn dadurch eine bedarfsgerechte Umsetzung ermöglicht wird und die Kernkomponenten der Intervention weiterhin vermittelt werden (Durlak \& DuPre, 2008). Da die Orientierung am Skript eine präzisere Einhaltung der Programmvorgaben vermuten lässt (Lane, Bocian, MacMillan, \& Gresham, 2004), ergibt sich für CReActivity kein eindeutiges Bild, inwieweit die vorgenommenen Veränderungen die Implementierungsqualität begünstigt oder verringert haben.

Die Analyse zur Implementierungshäufigkeit verdeutlichte, dass sich die Lehrerinnen bei der Interventionsdurchführung an den Rahmenbedingungen der Schulen orientieren mussten. Diesen konnten sich die Lehrerinnen größtenteils anpassen, jedoch führten zeitliche 
Beschränkungen zu einer unzureichend empfundenen Implementierungshäufigkeit, welche die Lehrerinnen an einer erfolgreichen Bewegungssteigerung zweifeln ließ. Zeitmangel stellt nicht nur ein Haupthindernis schulbasierter Interventionen zur Bewegungsförderung dar (Naylor et al., 2015), zudem könnten die Zweifel der Lehrerinnen die Implementierungsqualität beeinträchtigt haben, da Interventionsprogramme, die von den Vermittlern als effektiv wahrgenommen werden, meist eine höhere Implementierungsqualität aufweisen als ineffektiv wahrgenommene Programme (Domitrovich et al., 2008).

Hinsichtlich der Qualität der Programmweitergabe zeigte sich eine hohe Motivation und großes Interesse der Lehrerinnen zur Studienteilnahme. Dies kann die Implementierungsqualität begünstigen (Domitrovich et al., 2008). Jedoch kann hohes Interesse an einem Programm auch zu einer unverhältnismäßig positiven Bewertung führen (Sygusch \& Herrmann, 2009).

Der Einfluss des Lehrerengagements auf die Qualität der Programmweitergabe und auf das Sich-Involvieren der Schülerinnen wird unter Berücksichtigung der Schülerinnen-Interviews deutlich. Deren Wahrnehmung des Unterrichts ist durch die Lehrenden-Lernenden-Interaktion und das Engagement der Lehrerinnen geprägt. Miethling und Krieger (2004) schildern die Relevanz dieses Engagements für Schüler und Schülerinnen im Sportunterricht. Demnach wirkt geglücktes Engagement motivierend, während fehlendes Engagement demotiviert und bei gebrochenem Engagement die Bemühungen der Lehrkraft zwar erkannt aber nicht beachtet werden. Je nach wahrgenommenem Lehrerengagement sind die beschriebenen unterschiedlichen Verhaltensursachen der Schülerinnen somit nicht verwunderlich. Neben selbstbestimmteren Handlungsmotiven, wie Freude am Unterricht oder Lernbereitschaft, wurden auch externale Regulationsmechanismen, wie beispielweise die Vermeidung von negativen Konsequenzen, sichtbar. Vor dem Hintergrund der SBT deutet Letzteres eher auf eine Hemmung von AKS und damit auf potenzielle Einschränkungen der
Implementierungsqualität hin. Jedoch vermittelten die Lehrkräfte in Bezug auf das Involvieren der Teilnehmer ein positives Bild. Hohes Engagement der Schülerinnen und aktive Teilhabe am Sportunterricht überraschte die Lehrerinnen und vermittelte eine wahrgenommene Wirksamkeit. Die Wahrnehmung einer solchen Effektivität erhöht die Implementierungsqualität und wird für die CReActivity-Studie positiv gewertet (Domitrovich et al., 2008).

In der Analyse zur Anwendbarkeit der Intervention wurde die Einschätzung der Lehrerinnen deutlich, die Intervention besonders im späteren Verlauf umsetzen zu können. Diese positive Beurteilung der Fähigkeiten zur Interventionsumsetzung könnte die Implementierung begünstigt haben (Reeve et al., 2013). Studien bestätigen zudem den Erfahrungsgewinn im Interventionsverlauf und damit gesteigerte Fähigkeiten zur korrekten Programmimplementierung (Aelterman et al., 2013; Berkel, Mauricio, Schoenfelder, \& Sandler, 2011; Domitrovich et al., 2008). Zur Angemessenheit der Intervention zeigen sich zwei relevante Einschätzungen der Lehrerinnen. Einerseits kritisierten sie den TheoriePraxis-Konflikt, wonach die konzipierten Stunden nur unter optimalen Rahmenbedingungen durchführbar seien. Andererseits bewerteten sie die Intervention für die Zielgruppe insgesamt als angemessen. Durch die Erfahrungen der Lehrerinnen konnte der CReActivitySportunterricht an die Bedürfnisse der Schülerinnen und die schulischen Gegebenheiten angepasst werden, wodurch von einer kontextuellen Angemessenheit ausgegangen werden kann. Diese stellt einen wichtigen Faktor hoher Implementierungsqualität dar (Naylor et al., 2015). Somit zeigen sich Anpassungen hier als Möglichkeit bedarfsgerechter Implementierung, die potenziell mit erhöhter Teilnahme am Unterricht einhergeht (Berkel et al., 2011). Die kritische Beurteilung der Lehrerinnen bezüglich dieser notwendigen Anpassungen kann einen erneuten Hinweis auf Einschränkung der Implementierungsqualität darstellen. Jedoch kann diese Rückmeldung auch positiv gewertet werden, da erfahrene Lehrer Programme kritischer bewerte- ten als Unerfahrene, die Programme jedoch präziser implementieren (Aelterman et al., 2013; Berkel et al., 2011; Dusenbury, Brannigan, Hansen, Walsh, \& Falco, 2004).

Abschließend zeigt die Analyse zur Akzeptanz von CReActivity, dass die Lehrerinnen die Intervention insgesamt als bereichernd einschätzten. Diese hohe Akzeptanz und die damit einhergehende wahrgenommene Wirksamkeit konnte sich unter Umständen positiv auf die Implementierungsqualität auswirken (Domitrovich et al., 2008).

Zusammenfassend deuten die Ergebnisse zu Akzeptanz, Anwendbarkeit und Sich-Involvieren der Schülerinnen auf eine hohe Implementierungsqualität, besonders im späteren Interventionszeitraum hin. Dennoch lassen die Ergebnisse zur Qualität der Programmweitergabe und des Sich-Involvierens aus Schülerinnensicht lehrerbezogene Unterschiede vermuten. Einschränkungen der Implementierungsqualität sind hauptsächlich durch die Zweifel der Lehrerinnen an der Effektivität aufgrund der Implementierungshäufigkeit möglich. Inwieweit Anpassungen die Implementierungsqualität beeinflusst haben, kann anhand der vorliegenden Daten nicht geklärt werden.

In diesem Zusammenhang muss die Wahl der qualitativen Herangehensweise hinterfragt werden. Diese wurde gewählt, da sie in besonderem Maß kontextuelle Unterschiede und Perspektiven der Teilnehmer berücksichtigen kann, die bei der Überprüfung der Implementierung von Interventionen zur Förderung der körperlichen Aktivität im Schulsetting besonders relevant erscheint (Naylor et al., 2015). Einerseits ergab sich damit ein klarer Mehrwert hinsichtlich der Ergebnisse zur Beurteilung der Akzeptanz, Anwendbarkeit, des Sich-Involvierens und der Qualität der Programmweitergabe, indem die Einschätzungen der Teilnehmer überzeugend widergespiegelt werden konnten. Andererseits war aufgrund dieses subjektiven Charakters eine konkrete Beurteilung der Implementierungshäufigkeit nicht möglich. Hier lassen sich lediglich Rückschlüsse auf die resultierenden Konsequenzen schließen. Auch bei der Beurteilung der Einhaltung der 
Programmvorgaben stieß der qualitative Ansatz an Grenzen und konnte nicht gänzlich klären, in welcher Art und Weise die Interventionskomponenten verändert wurden. Für künftige Implementierungsinterventionen empfiehlt sich eine Kombination qualitativer und quantitativer Erhebungsmethoden. Diese Beobachtung deckt sich mit den Empfehlungen für Prozessevaluationen von Moore et al. (2015), nach denen qualitative Methoden insbesondere Erfahrungen und Einschätzungen der Intervention ermöglichen, die Einhaltung von Programvorgaben und die Implementierungshäufigkeit jedoch vorzugsweise anhand quantitativer Daten erhoben werden sollen.

Zudem besitzt die gewählte Methodik weitere Limitationen und Stärken. Zur methodischen Stärke der Untersuchung zählt das deduktiv-induktive Kodierverfahren, da es einen weitestgehend datengesteuerten Analyseprozess ohne Vernachlässigung theoretischer Überlegungen ermöglichte. Die Leitfadeninterviews erlaubten innerhalb eines leitenden Rahmens eine relativ flexible Gesprächsführung (Krieger, 2008). Die gewählten Face-to-face-Interviews konnten zudem detailliertere Ergebnisse hervorbringen, da ein individuellerer Gesprächsverlauf durch verbessertes Deuten nonverbaler Signale ermöglicht wurde (Novick, 2008).

Dennoch stellen Leitfadeninterviews eine indirekte Form der Implementierungsüberprüfung dar, die - verglichen mit direkten Methoden - zu höheren Bewertungen der Implementierungsqualität führen kann (Lane, Beebe-Frankenberger, Lambros, \& Pierson, 2001). AuBerdem lieferten Interviewteilnehmerinnen möglicherweise sozial erwünschte Antworten oder konnten sich an Situationen nicht detailliert erinnern, wodurch die Ergebnisse möglicherweise verfälscht wurden. Auch war die Standardisierung der Datenerhebung nur teilweise gegeben. Zeitdruck und ungeeignete Räumlichkeiten führten zu Verkürzungen oder Störungen der Interviews. Vertretungslehrkräfte ohne Bezug zur Klasse konnten möglicherweise nicht gemäß den Auswahlkriterien die Teilnehmerinnen auswählen. Weitere Einflüsse ergeben sich aus den Limitationen der TA. Es be- steht die Möglichkeit, dass verschiedene Betrachter die Aussagen der Interviewteilnehmer unterschiedlich interpretieren. Zudem ist die TA nur begrenzt in der Lage, Ungereimtheiten aufzudecken, da Aussagen zu einheitlichen Meinungen zusammengefasst werden (Braun \& Clarke, 2013). Vor dem Hintergrund dieser Meinungsbildung stellt die geringe Interviewanzahl eine potenzielle Beeinflussung der Ergebnisse dar.

\section{Schlussfolgerung}

Die Ergebnisse verweisen insgesamt auf eine heterogene, lehrerabhängige Implementierungsqualität. Im späteren Verlauf der CReActivity-Intervention kann von einer höheren Implementierungsqualität ausgegangen werden. Unklar bleibt die Auswirkung der Anpassungen der Lehrerinnen auf die Implementierungsqualität. Zukünftige Studien sollten eine präzise Dokumentation der Anpassungen realisieren und Lehrercharakteristika berücksichtigen, um eindeutige Schlüsse hinsichtlich der Implementierungsqualität ziehen zu können. Da die Lehrerinnen eine große Motivation hinsichtlich der Teilnahme und Durchführung der Studie vermittelten, könnte mit Hilfe eines entsprechenden Trainings die Implementierungsqualität zukünftiger Interventionen gesteigert werden.

\section{Korrespondenzadresse}

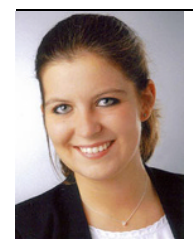

Denise Renninger

Fakultät für Sport- und Gesundheitswissenschaften, Technische Universität München

Georg-Brauchle-Ring 60/62, 80992 München, Deutschland denise.renninger@tum.de

Danksagung. Wir bedanken uns bei allen Schulen, Lehrerinnen und Schülerinnen, die an der Studie teilgenommen haben und bei der Deutschen Forschungsgesellschaft für die finanzielle Unterstützung.

Förderung. Das Forschungsprojekt wurde von der Deutschen Forschungsgemeinschaft gefördert (Projektnummer DE2680/3-1).

Author Contribution. Denise Renninger, David Sturm und Yolanda Demetriou trugen zur Konzeption und Gestaltung der Studie bei. Die Interviewleitfäden wurden von allen Autoren entwickelt. Die
Interviews und qualitativen Analysen wurden von Denise Renninger durchgeführt. Claus Krieger, David Sturm und Yolanda Demetriou unterstützten diesen Prozess. Alle Autoren trugen zur Interpretation der Ergebnisse bei. Den ersten Entwurf des Manuskripts verfasste Denise Renninger. Alle Autoren beurteilten und kommentierten frühere Versionen des Manuskripts. Yolanda Demetriou beaufsichtigte und erhielt finanzielle Mittel für die Studie. Alle Autoren haben das endgültige Manuskript gelesen und genehmigt.

Funding. Open Access funding enabled and organized by Projekt DEAL.

\section{Einhaltung ethischer Richtlinien}

Interessenkonflikt. D. Renninger, D. J. Sturm, C. Krieger und Y. Demetriou geben an, dass kein Interessenkonflikt besteht.

Alle beschriebenen Untersuchungen am Menschen oder an menschlichem Gewebe wurden mit Zustimmung der zuständigen Ethikkommission, im Einklang mit nationalem Recht sowie gemäß der Deklaration von Helsinki von 1975 (in der aktuellen, überarbeiteten Fassung) durchgeführt. Die Ethikkommission der Technischen Universität München genehmigte die Studie, registriert unter 155/16S. Das bayerische Staatsministerium für Unterricht und Kultus genehmigte das Forschungsvorhaben hinsichtlich Inhalten und Datenschutz. Schulleitung und Elternräte gaben eine schriftliche Einverständniserklärung zur Durchführung der Studie. Lehrer, Eltern und Kinder gaben eine schriftliche Einverständniserklärung zur Teilnahme an der Studie. Schüler und Lehrer gaben vor den Interviews eine mündliche Einverständniserklärung $\mathrm{ab}$.

Open Access. Dieser Artikel wird unter der Creative Commons Namensnennung 4.0 International Lizenz veröffentlicht, welche die Nutzung, Vervielfältigung, Bearbeitung, Verbreitung und Wiedergabe in jeglichem Medium und Format erlaubt, sofern Sie den/die ursprünglichen Autor(en) und die Quelle ordnungsgemäß nennen, einen Link zur Creative Commons Lizenz beifügen und angeben, ob Änderungen vorgenommen wurden.

Die in diesem Artikel enthaltenen Bilder und sonstiges Drittmaterial unterliegen ebenfalls der genannten Creative Commons Lizenz, sofern sich aus der Abbildungslegende nichts anderes ergibt. Sofern das betreffende Material nicht unter der genannten Creative Commons Lizenz steht und die betreffende Handlung nicht nach gesetzlichen Vorschriften erlaubt ist, ist für die oben aufgeführten Weiterverwendungen des Materials die Einwilligung des jeweiligen Rechteinhabers einzuholen.

Weitere Details zur Lizenz entnehmen Sie bitte der Lizenzinformation auf http://creativecommons.org/ licenses/by/4.0/deed.de.

\section{Literatur}

Aelterman, N., Vansteenkiste, M., Van Keer, H., De Meyer, J., Van den Berghe, L., \& Haerens, L. (2013). Development and evaluation of a training on need-supportive teaching in physical education: 
qualitative and quantitative findings. Teaching and Teacher Education, 29, 64-75. https://doi. org/10.1016/j.tate.2012.09.001.

Berkel, C., Mauricio, A. M., Schoenfelder, E., \& Sandler, I. N. (2011). Putting the pieces together: an integrated model of program implementation. Prevention Science, 12(1), 23-33. https://doi.org/ 10.1007/s11121-010-0186-1.

Braun, V., \& Clarke, V. (2006). Using thematic analysis in psychology. Qualitative research in psychology, 3(2), 77-101

Braun, V., \& Clarke, V. (2013). Successful qualitative research: a practical guide for beginners. London: SAGE.

Deci, E. L., \& Ryan, R.M. (2000). The "what" and "why" of goal pursuits: human needs and the self-determination of behavior. Psychological Inquiry, 11(4), 227-268. https://doi.org/10.1207/ S15327965PLI1104 01.

Deci, E. L., \& Ryan, R. M. (2002). Overview of selfdetermination theory: an organismic dialectical perspective. In E. L. Deci \& R.M. Ryan (Hrsg.), Handbook of self-determination research (S. 3-33). Rochester: University of Rochester Press.

Deci, E. L., \& Ryan, R. M. (2008). Facilitating optimal motivation and psychological well-being across life's domains. Canadian psychology/Psychologie canadienne, 49(1), 14. https://doi.org/10.1037/ 0708-5591.49.1.14.

Demetriou, Y., \& Bachner, J. (2019). A school-based intervention based on self-determination theory to promote girls' physical activity: study protoco of the CReActivity cluster randomised controlled trial. BMC Public Health, 19(1), 519. https://doi. org/10.1186/s12889-019-6817-y.

Demetriou, Y., Bucksch, J., Hebestreit, A., Schlund, A., Niessner, C., Schmidt, S. C. E., Reimers, A. K., et al. (2019). Germany's 2018 report card on physical activity for children and youth. German Journal of Exercise and Sport Research, 49(2), 113-126. https://doi.org/10.1007/s12662-019-00578-1.

Dobbins, M., Husson, H., DeCorby, K., \& LaRocca, R.L. (2013). School-based physical activity programs for promoting physical activity and fitness in children and adolescents aged 6 to 18 . Cochrane Database of Systematic Reviews. https://doi.org/ 10.1002/14651858.CD007651.pub2.

Domitrovich, C. E., Bradshaw, C. P., Poduska, J. M., Hoagwood, K., Buckley, J. A., Olin, S., lalongo, N.S., et al. (2008). Maximizing the implementation quality of evidence-based preventive interventions in schools: a conceptual framework. Advances in School Mental Health Promotion, 1(3), 6-28. https://doi.org/10.1080/1754730X. 2008.9715730

Durlak, J.A. (2016). Programme implementation in social and emotional learning: basic issues and research findings. Cambridge Journal of Education, 46(3), 333-345. https://doi.org/10 1080/0305764X.2016.1142504.

Durlak, J. A., \& DuPre, E.P. (2008). Implementation matters: a review of research on the influence of implementation on program outcomes and the factors affecting implementation. American Journal of Community Psychology, 41(3), 327. https://doi.org/10.1007/s10464-008-9165-0.

Dusenbury, L., Brannigan, R., Hansen, W. B., Walsh, J., \& Falco, M. (2004). Quality of implementation: developing measures crucial to understanding the diffusion of preventive interventions. Health Education Research, 20(3), 308-313. https://doi. org/10.1093/her/cyg134.
Finger, J.D., Varnaccia, G., Borrmann, A., Lange, C., \& Mensink, G. (2018). Körperliche Aktivität von Kindern und Jugendlichen in Deutschland Querschnittergebnisse aus KiGGS Welle 2 und Trends. Journal of Health Monitoring. https://doi. org/10.17886/RKI-GBE-2018-006.2.

Hallal, P.C., Victora, C. G., Azevedo, M. R., \&Wells, J. C. K. (2006). Adolescent physical activity and health. Sports medicine, 36(12), 1019-1030. https://doi. org/10.2165/00007256-200636120-00003.

Krieger, C. (2008). Leitfaden-Interviews. In W.D. Miethling \& M. Schierz (Hrsg.), Qualitative Forschungsmethoden in der Sportpädagogik (S. 45-63). Schorndorf:Hofman-Verlag.

Lane, K. L., Beebe-Frankenberger, M.E., Lambros, K. M., \& Pierson, M. (2001). Designing effective interventions for children at-risk for antisocial behavior: An integrated model of components necessary for making valid inferences. Psychology in the Schools, 38(4), 365-379. https://doi. org/10.1002/pits.1025.

Lane, K. L., Bocian, K. M., MacMillan, D. L., \& Gresham, F. M. (2004). Treatment integrity: an essential-but often forgotten-component of schoolbased interventions. Preventing School Failure: Alternative Education for Children and Youth, 48(3),36-43. https://doi.org/10.3200/PSFL.48.3. 36-43.

Lonsdale, C., Rosenkranz, R. R., Peralta, L. R., Bennie, A., Fahey, P., \& Lubans, D. R. (2013). A systematic review and meta-analysis of interventions designed to increase moderate-to-vigorous physical activity in school physical education lessons. Preventive Medicine, 56(2), 152-161. https://doi.org/10.1016/j.ypmed.2012.12.004.

Miethling, W.-D., \& Krieger, C. (2004). Schüler im Sportunterricht. Die Rekonstruktion relevanter Themen und Situationen des Sportunterrichts aus Schülersicht (RETHESIS). Bd. 140. Schorndorf: Verlag KarlHofmann.

Moore, G. F., Audrey, S., Barker, M., Bond, L., Bonell, C., Hardeman, W., Baird, J., et al. (2015). Process evaluation of complex interventions: medical research council guidance. $B M J$ : British Medical Journal, 350, h1258. https://doi.org/10.1136/ bmj.h1258.

Naylor, P.-J., Nettlefold, L., Race, D., Hoy, C., Ashe, M. C., Wharf Higgins, J., \& McKay, H. A. (2015). Implementation of school based physical activity interventions: a systematic review. Preventive Medicine, 72, 95-115. https://doi.org/10.1016/j. ypmed.2014.12.034.

Novick, G. (2008). Is there a bias against telephone interviews in qualitative research? Research in Nursing \& Health, 31(4), 391-398. https://doi. org/10.1002/nur.20259.

Ntoumanis, N., Quested, E., Reeve, J., \& Cheon, S.H. (2018). Need supportive communication: Implications for motivation in sport, exercise, and physical activity. In B. Jackson, J. A. Dimmock \& J. Compton (Hrsg.), Persuasion and communication in sport, exercise, and physical activity (S. 155-169). Abingdon: Routledge.

Poitras, V.J., Gray, C.E., Borghese, M.M., Carson, V., Chaput, J.-P., Janssen, I., Tremblay, M.S., et al. (2016). Systematic review of the relationships between objectively measured physical activity and health indicators in school-aged children and youth. Applied Physiology, Nutrition, and Metabolism, 41(6), 197-239. https://doi.org/10. 1139/apnm-2015-0663.

Reeve, J. (2009). Why teachers adopt a controlling motivating style toward students and how they can become more autonomy supportive.
Educational Psychologist, 44(3), 159-175. https:// doi.org/10.1080/00461520903028990.

Reeve, J. (2016). Autonomy-supportive teaching: what it is, how to do it. In W.C. Liu, J.C. K. Wang \& R. M. Ryan (Hrsg.), Building autonomous learners: perspectives from research and practice using self-determination theory (1. Aufl. S. 129-152). Berlin: Springer. https://doi.org/10.1007/978981-287-630-0_7.

Reeve, J., Vansteenkiste, M., Assor, A., Ahmad, I. Cheon, S. H., Jang, H., Wang, C. K. J., et al. (2013). The beliefs that underlie autonomy-supportive and controlling teaching: A multinational investigation. Motivation and Emotion, 38(1), 93-110. https://doi.org/10.1007/s11031-0139367-0.

Strong, W. B., Malina, R. M., Blimkie, C. J. R., Daniels, S. R., Dishman, R. K., Gutin, B., Trudeau, F., et al. (2005). Evidence based physical activity for school-age youth. The Journal of Pediatrics, 146(6), 732-737. https://doi.org/10.1016/j.jpeds.2005.01.055.

Sygusch, R., \& Herrmann, C. (2009). Entwicklungsförderung im außerschulischen Kinder- und Jugendsport. Sportwissenschaft, 39(3), 210-222. https://doi.org/10.1007/s12662-009-0061-z.

Sygusch, R., Bähr, I., Gerlach, E., \& Bund, A. (2013). Orientierungspunkte einer Programmevaluation in der Sportpädagogik. Zeitschrift für sportpädagogische Forschung, 1(1), 31-54.

Telama, R. (2009). Tracking of physical activity from childhood to adulthood: a review. Obesity Facts, 2(3), 187-195. https://doi.org/10.1159/ 000222244

Vasconcellos, D., Parker, P.D., Hilland, T., Cinelli, R., Owen, K. B., Kapsal, N., Ryan, R. M., et al. (2019). Self-determination theory applied to physical education: A systematic review and metaanalysis. Journal of Educational Psychology. https://doi.org/10.1037/edu0000420.

Wolters, P., Klinge, A., Klupsch-Sahlmann, R., \& Sinning, S. (2009). Was ist nach unseren Vorstellungen guter Sportunterricht? Sportunterricht, 58(5), 67-72.

World Health Organization (2010). World Health Organization. World Health Organization: WHO Press. 